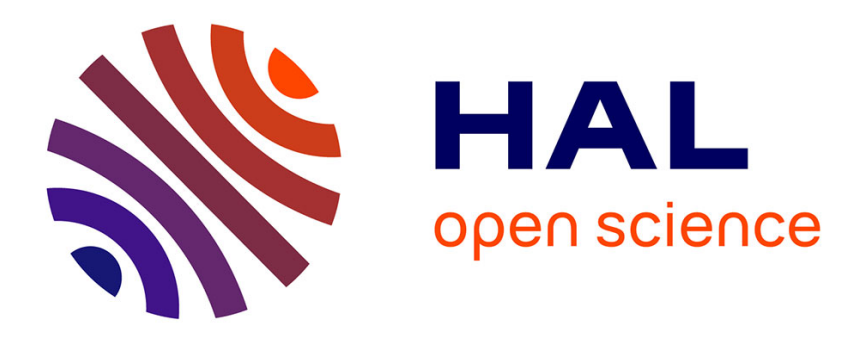

\title{
Interface entropy and phase transitions in frustrated ising systems
}

\author{
B. Derrida, J.M. Maillard, J. Vannimenus, S. Kirkpatrick
}

\section{To cite this version:}

B. Derrida, J.M. Maillard, J. Vannimenus, S. Kirkpatrick. Interface entropy and phase transitions in frustrated ising systems. Journal de Physique Lettres, 1978, 39 (23), pp.465-468. 10.1051/jphyslet:019780039023046500 . jpa-00231546

\section{HAL Id: jpa-00231546 https://hal.science/jpa-00231546}

Submitted on 1 Jan 1978

HAL is a multi-disciplinary open access archive for the deposit and dissemination of scientific research documents, whether they are published or not. The documents may come from teaching and research institutions in France or abroad, or from public or private research centers.
L'archive ouverte pluridisciplinaire HAL, est destinée au dépôt et à la diffusion de documents scientifiques de niveau recherche, publiés ou non, émanant des établissements d'enseignement et de recherche français ou étrangers, des laboratoires publics ou privés. 


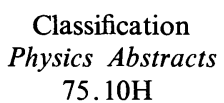

\title{
INTERFACE ENTROPY AND PHASE TRANSITIONS IN FRUSTRATED ISING SYSTEMS
}

\author{
B. DERRIDA \\ Institut Laue-Langevin, 38042 Grenoble Cedex, France \\ J. M. MAILLARD, J. VANNIMENUS \\ Groupe de Physique des Solides, Ecole Normale Supérieure, \\ 75231 Paris Cedex 05, France \\ and S. KIRKPATRICK $(*)$ \\ I.B.M., T.J. Watson Research Laboratory, Yorktown Heights, \\ P.O. Box 218, New York 10598, U.S.A.
}

(Reçu le 13 septembre 1978, accepté le 13 octobre 1978)

\begin{abstract}
Résumé. - L'annulation de l'énergie d'interface à $T=0$ est compatible avec l'existence d'une transition de phase à température finie, dans des systèmes de spins d'Ising. Dans des systèmes frustrés, la création d'une interface peut coûter de l'entropie. Cet effet peut suffire à entraîner une température de transition non nulle. Nous donnons un exemple explicite sur un modèle périodique et discutons quelques conséquences possibles pour les verres de spins d'Ising en deux dimensions.

Abstract. - It is pointed out that the vanishing of the interface energy at $T=0$ is compatible with the existence of a phase transition at finite temperature in Ising systems. In frustrated systems an interface may cost entropy. This effect may be sufficient to produce a non-zero transition temperature. We give an explicit example of this behaviour on a periodic model. Some possible consequences for 2-D Ising spin-glasses are discussed.
\end{abstract}

Recent theories of the transition from ferromagnet to spin-glass [1], and of the lower critical dimensionality of the spin-glass phase [2], have drawn attention to the properties of interfaces - domain walls - in these systems.

In pure and in weakly disordered Ising systems [3], the transition temperature is also the temperature at which the interface free energy vanishes. Vannimenus and Toulouse [1] have associated the vanishing of the interface energy, at $T=0$, with the disappearance of ferromagnetic order for a critical concentration $x_{c}$ (the frustration threshold) of antiferromagnetic interactions in an otherwise ferromagnetic two-dimensional Ising model.

Reed, Moore and Bray [2] have taken this suggestion further, arguing that a zero defect energy would

(*) This work was performed while the author was a visiting professor at the University Paris VI. prevent the occurrence of any long-range order, even of the Edwards-Anderson type. From their numerical studies of the interface energies in spin-glass models, they have argued that in 2 and 3 dimensions the paramagnetic phase would persist down to zero temperature at these concentrations where the ferromagnetic phase is unstable [4].

Their argument in its simplest form may be phrase as follows. If one considers two spins in a system at $T=0$, and there is a possibility of creating an interface with zero energy cost between the two spins, then for each configuration of the system in which the two spins are parallel there will be a configuration of equal energy in which the two spins are antiparallel. Thus there cannot be any correlation between the two spins and by extension, no long-range order.

There is a flaw in this argument. It is not necessarily true that the number of configurations with the spin parallel is equal to the number of configurations with the spins antiparallel. Consider the correlations 
between spins 1 and 2 in figure 1 . If these spins are antiparallel, the bond at the bottom of the figure is not satisfied and can be viewed as a defect. If spins 1 and 2 are parallel, either one of the upper bonds is not satisfied. Thus the defect can occur in two positions,

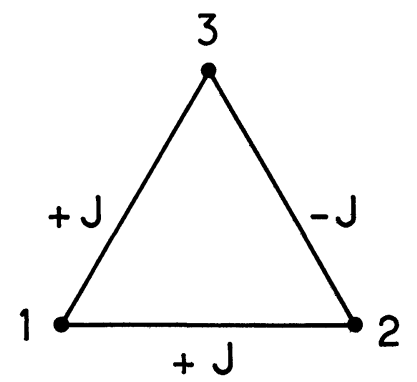

FIG. 1. - Ising spins on a frustrated triangle.

and $S_{1} S_{2}=+1$ is twice as likely as $S_{1} S_{2}=-1$. The expectation value $\left\langle S_{1} S_{2}\right\rangle$ therefore is $+1 / 3$, not 0 , even though there is no energy difference between the three possible positions of the defect. The difference in degeneracy between $S_{1} S_{2}=+1$ and $S_{1} S_{2}=-1$ can be viewed as the entropy cost of placing a defect between $S_{1}$ and $S_{2}$.

The same kind of reasoning applies to the correlations between any pair of spins in a system where the defect energy vanishes. It extends to the case where the defect energy is bounded for large defect linear dimensions, and Reed et al.'s argument for this case suffers from the same flaw of neglecting degeneracy differences.

We give in this letter a general formulation for the free energy of a defect in a frustrated system, stressing the importance of entropy effects. As an illustration, we study a periodic model for which the interface energy vanishes at $T=0$ but the interface entropy is non-zero. For this model there is a phase transition at finite $T$. Finally we discuss some implications of these arguments for Ising spin-glasses.

1. General formulation. - In a system where all interactions are ferromagnetic, the definition of a defect and its free energy is no problem. At low temperatures, the system is ordered and the boundary conditions that minimize the total energy are those compatible with this order, i.e. all spins on the boundary pointing in the same direction. Let us denote this choice condition A. A defect (a domain wall), is created by reversing the direction of the boundary spins in one half of the system (condition B). The defect free energy is then just the difference between the free energies :

$$
\sigma(T)=F_{\mathbf{B}}(T)-F_{\mathbf{A}}(T)
$$

$\sigma(T)$ is proportional to the length of the interface so created at low temperatures and vanishes at $T_{\mathrm{c}}$.

For a system with interactions of both signs, condition A will not necessarily remain the most favourable one (just consider a chain with one negative bond). If the bond distribution has no frustration, it is still possible to find the best boundary conditions, just by redefining every spin variable locally step by step. But in a model with frustration there may be no unique way to perform such a redefinition. We then have to consider all possible boundary conditions and select one of the configurations that minimize the energy as the reference condition $A^{\prime}$. If there remain several possibilities corresponding to different ordered states, we have to select the most probable one that maximizes the entropy. To define a defect we play the same game as before and reverse the boundary spins in one half of the system, starting from $\mathrm{A}^{\prime}$, to obtain condition $\mathrm{B}^{\prime}$.

The defect free energy is now defined as :

$$
\begin{aligned}
\sigma(T) & =F_{\mathrm{B}^{\prime}}(T)-F_{\mathrm{A}^{\prime}}(T) \\
& \sim E_{\mathrm{B}^{\prime}}-E_{\mathrm{A}^{\prime}}+T\left(S_{\mathrm{A}^{\prime}}-S_{\mathrm{B}^{\prime}}\right)
\end{aligned}
$$

at low $T$, where $E_{\mathrm{A}^{\prime}}$ and $S_{\mathrm{A}^{\prime}}$ are the $T=0$ values of energy and entropy. If $E_{\mathrm{B}^{\prime}}$ is superior to $E_{\mathrm{A}^{\prime}}$, the defect free energy behaves qualitatively like the interface free energy in the ferromagnetic case. However, if the defect energy per unit length vanishes at $T=0$, the entropy term may become dominant and give a positive contribution at low $T$. The interface may be considered to have negative entropy in that case, though naturally the complete system has positive entropy. At higher temperatures, $\sigma$ decreases and it vanishes at a certain $T_{\mathrm{c}}$ where a phase transition occurs.

Such behaviour is reminiscent of rubber, for which strong entropy effects oppose the energy terms and lead to unconventional thermodynamic properties [5]. Usually a defect is defined relatively to a state favourable in energy but of low statistical weight; here, as many states may be favourable in energy, the reference state becomes the most probable one and this leads to a defect free energy increasing with temperature.

2. An explicit periodic example. - Let us now work out an explicit example of order due to entropy. The model we consider has been recently introduced by André, Bidaux, Carton, Conte and De Seze [6] in the study of frustrated systems ; it is a very interesting generalization of a model introduced by Villain [7], the odd model.

In the odd model, all bonds have the same magnitude and the bonds in every second column are antiferromagnetic, so that all elementary squares (plaquettes) are frustrated. Here these bonds have a strength $\left(-J^{\prime}\right)$ larger in absolute value than the strength $J$ of the positive bonds (Fig. 2). All plaquettes are still frustrated but the number of ground states is much reduced (the entropy at $T=0$ is non-zero but lower than for the odd model). It is energetically favourable to frustrate the weakest bonds and keep antiferromagnetic order on the negative columns.

André et al. have made a detailed study of the ther- 

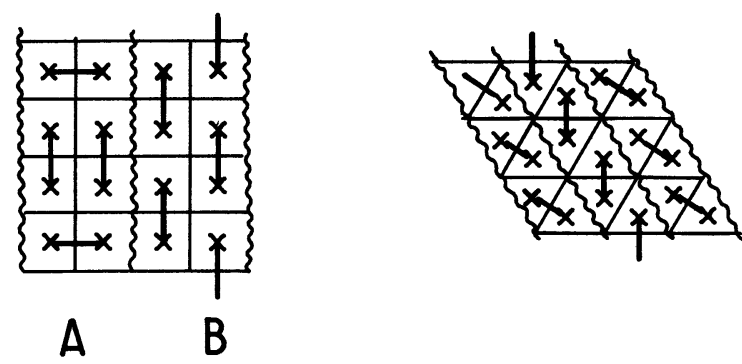

I

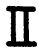

FIG. 2. - Two completely frustrated models with very different behaviour. In model I (the suspender model), the wiggled lines represent antiferromagnetic bonds of strength $-J^{\prime}$, the other bonds are ferromagnetic with strength $J$; if $J^{\prime}>J$, this model has a transition at finite temperature and it costs entropy to create an interface. In model II, all bonds are antiferromagnetic; if the bonds on the wiggled lines are the strongest $\left(J^{\prime}>J\right)$, no transition exists, and an interface costs no entropy. In both cases, the crosses represent the centres of the frustrated plaquettes, and the heavy lines intersect the frustrated bonds in a ground-state.

modynamic properties of this model, which they call the suspender model. Using both the Pfaffian method and the transfer matrix approach, they have shown that for $J^{\prime}>J$ the system has a transition at a finite $T_{\mathrm{c}}$, with $T_{\mathrm{c}}$ vanishing for $J^{\prime}=J$.

From the transfer matrix eigenvalues we can obtain the interface energy, following Watson [8], and it is all we need for the present purpose. The result depends on the direction considered and reads, after some algebra :

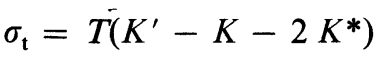

$\sigma_{1}=T\left[\sinh ^{-1}\left(\cosh 2 K \sinh \left(K^{*}-K^{* *}\right)\right)-\right.$

$$
\left.-K^{*}-K^{*}\right] \text {. }
$$

In these expressions, $\sigma_{\mathrm{t}}$ is the interface tree energy per unit length in a direction perpendicular to the antiferromagnetic chains and $\sigma_{1}$ the free energy for an interface parallel to the chains. $K$ stands for $J / T, K^{\prime}$ for $J^{\prime} / T$ and the starred quantities are the usual dual couplings :

$$
K^{*}=-1 / 2 \log (\tanh K) .
$$

The expression for $\sigma_{\mathrm{t}}$ is remarkably simple and similar to the form of the interface energy for the pure system. It can in fact be obtained directly by considering a restricted set of interface configurations, using the method of Müller-Hartmann and Zittartz [9], but like these authors we cannot prove why this procedure gives the correct result.

A graph of the interface energies is shown in figure 3 for the choice $J^{\prime}=3 J$. It is easy to check directly that both vanish at the same $T_{\mathrm{c}}$ as expected, and that $\sigma_{1}$ vanishes at $T=0$ with a finite slope. Explicitly one obtains in this limit :

$$
\sigma_{1} \sim T \log \left(\frac{1+\sqrt{5}}{2}\right) .
$$

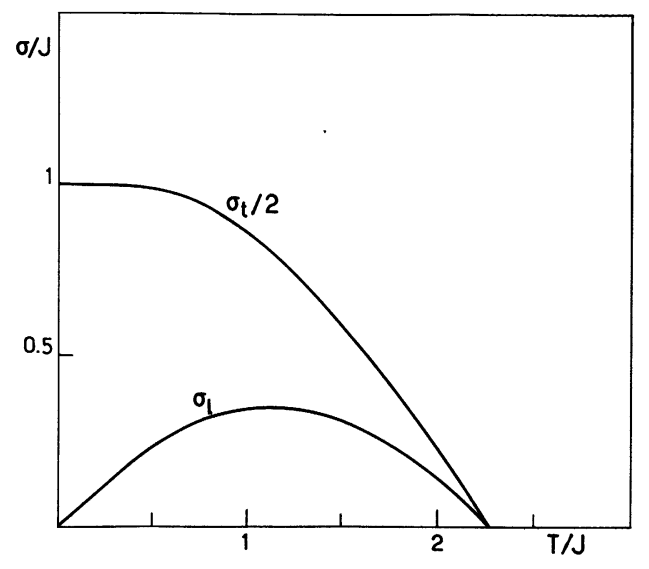

FIG. 3. - Plot of interface free energies versus temperature for the suspender model, with $J^{\prime}=3 J . \sigma_{\mathrm{t}}$ corresponds to an interface perpendicular to the antiferromagnetic chains (the wiggled lines of Fig. 2I), and behaves as in the pure Ising model. $\sigma_{1}$ corresponds to an interface parallel to the chains and vanishes at $T=0$, as shown by eq. (5).

This result is independent of $J^{\prime} / J$ and may be interpreted very simply. One can see on figure 2 that if arrangement B exists somewhere in the suspender, it persists everywhere (for a ground-state) and the frustrated bonds are known for all the suspender. This arrangement corresponds to out-of-phase antiferromagnetic chains and has a twofold degeneracy. In case A, at every level of the suspender a choice exists : if at one level no bond is frustrated, at the next level either no bond or two bonds are frustrated. But if two bonds are frustrated, at the next level no bond may be frustrated. Arrangement A corresponds to antiferromagnetic chains in phase, and is much more probable. One can calculate its entropy for one suspender :

$$
\log W_{\mathrm{A}} \sim n \log \left(\frac{1+\sqrt{5}}{2}\right)
$$

where $n$ is the number of horizontal rows.

From the considerations given above, one sees that the state without interface is the state where all the suspenders are in arrangement A. To create an interface, one has to replace arrangement $\mathrm{A}$ by arrangement $B$ in one of the suspenders. This does not change the energy of the system but it costs entropy, and one can say that the interface entropy is negative and reads :

$$
S_{\mathrm{I}}=-n \log \left(\frac{1+\sqrt{5}}{2}\right)
$$

In this way one recovers result (5) for the interface free energy at low temperatures.

The interface energy in the perpendicular direction does not vanish at zero $T$ but this in itself is not sufficient to produce a finite $T_{\mathrm{c}}$, as may be seen from other simple examples. Consider for instance model II of figure 2 [10]. In this triangular completely frustrated 
model, a phase transition exists only when the two equal negative couplings $(-J)$ are stronger than the third one $\left(-J^{\prime}\right)$, and the transition temperature can be deduced from an expression given by Syozi [11] :

$$
\sinh \left(\frac{2 J}{T_{\mathrm{c}}}\right)=\exp \left(\frac{2 J^{\prime}}{T_{\mathrm{c}}}\right)
$$

The drawing in figure 2 corresponds to the opposite situation $\left(J^{\prime}>J\right)$. In that case no transition subsists, though the entropy per spin vanishes at $T=0$, and though the interface energy in a direction transverse to the antiferromagnetic $J^{\prime}$ chains does not vanish at $T=0$. Such behaviour is in direct contrast to what happens in the suspender model, and we ascribe it to the absence of an interface entropy. It is easy to see that the two possible ground-states for every frustrated strip have the same probability, so reversing the spins in one half of the system costs neither energy nor entropy.

In conclusion, for this class of frustrated systems, the existence of a phase transition is not related to the value of the bulk entropy at $T=0$ (i.e. the total number of different ground-states), but to the existence of a negative interface entropy (i.e. a difference in the degeneracy of the possible ordered states).

3. Disordered systems. - The general discussion given above is valid for disordered materials. Once the frustration network is sufficiently developed, the defect energy (per unit length) for a system of $N \times N$ spins vanishes $[1,2]$, and the boundary conditions should be chosen to maximize the entropy (we consider models with a residual entropy, like the $\pm J$ model). If the entropy difference when a defect is created is proportional to $N$, a transition may occur at finite $T$.

We cannot prove directly that this kind of effect exists in spin-glasses, even for the simple two-dimensional model with $\pm J$ bonds. The Monte-Carlo methods are $a$ priori better suited to the problem. In practice, however, the bulk entropy is more difficult to obtain than the bulk energy, and the determination of a defect entropy is not a simple numerical task.
Moreover, free boundary conditions introduce extra size effects.

Therefore, we have studied a few random $(18 \times 18)$ samples directly, by hand, comparing the groundstates for different boundary conditions (periodic, antiperiodic on two sides and on all four sides). As expected, the ground-state energies are equal (within $4 J$ ) when enough frustration is present. What is less expected is that for given boundary conditions a majority of spins belongs to one ordered cluster and they always have the same relative orientation, so the correlations between the ground-states are quite strong. Suppose now that the periodic boundary conditions correspond to the most probable of these local orders. If the sample were infinite, with the same bond pattern periodically repeated, its most probable ground-states would consist of a patchwork - of local ground-states respecting the periodicity. Thus an infinite ordered cluster would exist at low temperatures, and a transition could occur at finite $T$ because of the entropy effect, just as in the suspender model.

The samples studied are too small to draw a final conclusion. Simulations on large samples will be necessary to obtain reliable results on the $N$-dependence of the interface entropy, and to see if this quantity plays an important part in Ising spin-glasses.

In this letter we have shown that the vanishing of the defect energy at $T=0$ does not imply the absence of a phase transition at finite temperatures. This point may be important for Ising spin-glasses above the frustration threshold. The example of a periodic system that we have studied is not general enough to be considered as an argument in favour of the existence of a phase transition in these spin-glasses, but it constitutes a useful counter example to keep in mind.

Acknowledgments. - Its interest was made clear to us by discussions with several colleagues. We particularly thank G. André, C. Bidaux, J. P. Carton, R. Conte and L. De Seze for introducing us to their suspender model and communicating their results before publication.

\section{References}

[1] Vannimenus, J. and Toulouse, G., J. Phys. C 10 (1977) L537.

[2] Reed, P., Moore, M. A. and Bray, A. J., J. Phys. C 11 (1978) L139.

[3] Fisher, M. E., J. Phys. Soc. Japan 26 Suppl. 87 (1969).

[4] Bray, A. J., Moore, M. A. and Reed, P., J. Phys. C 11 (1978) 1187.

[5] EdWards, S. F., Statistical Mechanics of Rubber, in « Polymer Networks », A. J. Chompff and S. Newman eds. (New York, Plenum Press) 1971.

[6] André, G.. Bidaux, R., Carton, J. P., Cọnte, R. and de Seze, L., submitted to $J$. Physique. These authors have adopted the name piled-up dominoes rather than suspender model for their model described in figure 2-I.

[7] Villain, J., J. Phys. C 10 (1977) 1717.

[8] Watson, P. G., in Phase Transitions and Critical Phenomena, C. Domb and M. S. Green eds. (Acad. Press) 1972, Vol. II, p. 112

[9] Müller-Hartmann, E. and Zittartz, J., Z. Phys. B 27 (1977) 261 ; Burkhardt, T. W., Z. Phys. B 29 (1978) 129 ; Southern, B. W., Z. Phys. B 30 (1978) 61.

[10] This example was suggested by Dr. SHERrington, D.

[11] Syozi, I., in Domb and Green, Vol. I, p. 322 (1972). 